\title{
Chronisch immunvermittelte Neuropathien - Diagnostik und Therapie
}

Autoren

Alexander Grimm, Hubertus Axer

\begin{abstract}
Die Diagnose einer immunvermittelten Neuropathie ist eine der wichtigsten Differenzialdiagnosen bei Polyneuropathien. Chronisch inflammatorische Neuropathien haben oft eine schwere neurologische Beeinträchtigung zur Folge, weswegen eine frühzeitige Diagnose zur Einleitung einer antientzündlichen Therapie essenziell ist. Therapiekonzepte beinhalten je nach Pathologie Immunglobuline, Kortikosteroide oder Plasmaaustauschverfahren.
\end{abstract}

\begin{tabular}{|c|c|}
\hline \multicolumn{2}{|c|}{ ABKÜRZUNGEN } \\
\hline CIDP & $\begin{array}{l}\text { chronisch inflammatorische demyeli- } \\
\text { nisierende Polyradikuloneuropathie }\end{array}$ \\
\hline CSA & $\begin{array}{l}\text { cross-sectional area; Querschnitt im } \\
\text { Ultraschall }\end{array}$ \\
\hline DADS & $\begin{array}{l}\text { distal acquired demyelinating sym- } \\
\text { metric neuropathy }\end{array}$ \\
\hline EFNS & $\begin{array}{l}\text { European Federation of Neurological } \\
\text { Societies }\end{array}$ \\
\hline MADSAM & $\begin{array}{l}\text { multifocal acquired demyelinating } \\
\text { sensory and motor neuropathy }\end{array}$ \\
\hline MGUS & $\begin{array}{l}\text { monoklonale Gammopathie unklarer } \\
\text { Signifikanz }\end{array}$ \\
\hline MMN & mutifokale motorische Neuropathie \\
\hline MSAP & motorisches \\
\hline & Summenaktionspotenzial \\
\hline NLG & Nervenleitgeschwindigkeit \\
\hline NSVN & nonsystemic vasculitic neuropathy \\
\hline POEMS & $\begin{array}{l}\text { Akronym für Polyneuropathie, Orga- } \\
\text { nomegalie, Endokrinopathie, mono- } \\
\text { klonale Gammopathie und } \\
\text { Hautveränderungen (Skin) }\end{array}$ \\
\hline SNAP & sensibles Nervenaktionspotenzial \\
\hline
\end{tabular}

\section{Einleitung}

Die Häufigkeit von immunvermittelten Neuropathien beträgt ca. 6-8 auf 100000 Menschen. Die häufigste Form ist die chronisch inflammatorische demyelinisierende Polyradikuloneuropathie (CIDP) sowie ihre Varianten [1, 2]. Des Weiteren finden sich paraproteinämische Neuropathien, die rein motorische mutifokale motorische Neuropathie (MMN) u.a. Diese Erkrankungen betreffen insgesamt Individuen jedweden Alters, vor allem jedoch Männer mittleren oder höheren Alters, insbesondere bei der MMN (Verhältnis 3:1).

All diesen Formen ist der überwiegend demyelinisierende Pathomechanismus gemein. Eine kürzlich beschriebene Unterform der CIDP, die Paranodopathie, zeigt oft eine eher axonale Schädigung. Darüber hinaus gibt es axonal betonte immunvermittelte Polyneuropathien, v. a. die vaskulitische Polyneuropathie.

In dieser Übersicht wollen wir uns auf die häufigen chronisch inflammatorischen Neuropathien mit großer klinischer Relevanz fokussieren:

- CIDP und ihre Varianten

- paraproteinämische Neuropathien

- MMN

- vaskulitische Neuropathien

Dabei hat es in den letzten Jahren Fortschritte im Bereich der Bildgebung (Nervenultraschall und MRT) gegeben, die in der Differenzialdiagnostik von chronischen Polyneuropathien nützliche Hinweise geben können.

\section{Chronisch inflammatorische demyelinisierende Polyradikuloneuropathie (CIDP)}

\section{Klinische Präsentation}

Die „klassische“ CIDP zeigt einen chronisch-progredienten oder schubförmigen Verlaufstyp über mindestens 2 Monate mit distal-symmetrischen Sensibilitätsstörungen, Parästhesien, proximal sowie distal beinbetonten Paresen und abgeschwächten oder fehlenden Muskeleigenreflexen 


\section{FALLBEISPIEL 1}

Eine 68-jährige Patientin stellt sich mit seit 4 Jahren progredienten aufsteigenden Dysästhesien der Füße und Unterschenkel vor. Seit 1 Jahr bereite ihr das Laufen und Treppensteigen zunehmend Beschwerden.

In der elektrophysiologischen Untersuchung finden sich ausgefallene SNAP des N. medianus und suralis, die motorische Nervenleitgeschwindigkeit (NLG) ist im N. tibialis mit $30 \mathrm{~m} / \mathrm{s}$ reduziert, die MSAP-Amplitude mit 2,6 mV distal und 2,2 mV proximal ebenso. Die F-Wellen sind nicht erhältlich. Die motorische NLG des N. medianus ist mit $15 \mathrm{~m} / \mathrm{s}$ ebenso deutlich reduziert, die MSAP-Amplitude mit 4,6 mV distal leicht und mit $1,6 \mathrm{mV}$ proximal deutlich reduziert.

Die Kriterien eines inkompletten Leitungsblocks sind bei deutlicher temporaler Dispersion nicht erfüllt. Die MSAP-Dauer ist mit 6,7 ms verlängert. Die F-Wellen-Persistenz ist mit $90 \%$ regelrecht, die F-M-Latenz mit 34,3 ms leicht verlängert ( $>$ Abb. 1). Im Liquor finden sich regelrechte Werte für Zellzahl, Glukose und Laktat, das Eiweiß ist mit $87 \mathrm{mg} / \mathrm{dl}$ erhöht. Differenzialdiagnostische Laboruntersuchungen sind unauffällig, im MRT finden sich T2-Hyperintensitäten der lumbalen Wurzeln mit leichter KM-Aufnahme

( Abb. 1). Im Ultraschall finden sich ubiquitär inhomogene Nervenschwellungen ( $\triangleright \mathbf{A b b} .1 \mathrm{~N}$. medianus am Oberarm mit einer Querschnittsfläche, CSA von $23 \mathrm{~mm}^{2}$, Normwert $<13 \mathrm{~mm}^{2}$ ).

Das Beispiel zeigt eine klassische Form der CIDP.

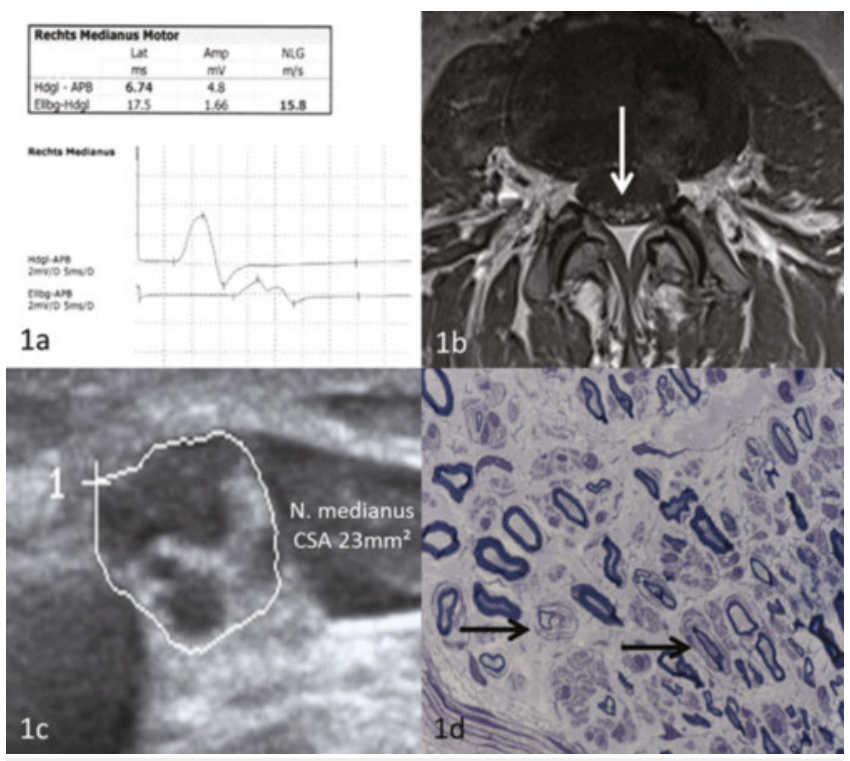

-Abb. 1 CIDP. Befunde der Zusatzdiagnostik; a CIDP mit demyelinisierenden Veränderungen in der Neurografie; b T2-Hyperintensitäten der Kaudafasern (weißer Pfeil) im MRT der LWS; c Proximale Nervenschwellungen im N. medianus im Ultraschall; $\mathbf{d}$ Ergebnisse einer Suralisnervbiopsie, u.a. mit Zwiebelschalen (schwarze Pfeile) als Zeichen der chronischen De- und Remyelinisierung.
[1]. In einigen Fällen kommt ein monophasischer, auch subakut beginnender Verlaufstyp vor, der mitunter schwierig von einem Guillain-Barré-Syndrom (GBS) zu unterscheiden ist, gerade bei Patienten, deren Symptomentwicklung zwischen 4 und 8 Wochen beträgt.

\section{Merke}

Beim GBS kann eine sekundäre Verschlechterung nach nachlassendem Effekt der Immuntherapie (treatment related deterioration) schwierig von einem 2. Schub einer CIDP zu unterscheiden sein [2, 3].

Die Demyelinisierungen bei der klassischen CIDP sind proximal betont (Wurzeln, Plexus, proximale Nervensegmente) und multilokulär. Allerdings finden sich zahlreiche Varianten (ca. $50 \%$ der Fälle) mit überwiegend motorischem („pure motor, CIDP“), sensorischem (distal acquired demyelinating symmetric neuropathy, DADS, oder bei Affektion der Hinterwurzeln „pure sensory, CISP“ mit pathologischen sensorisch evozierten Potenzialen und erhaltenen SNAP [4]) oder asymmetrischem Verlauf (Lewis-Sumner-Syndrom oder multifocal acquired demyelinating sensory and motor neuropathy, MADSAM). Auch Hirnnerven können betroffen sein, v. a. bei der MADSAM.

Je chronifizierter die Erkrankung, desto uneindeutiger werden klinischer Verteilungstyp, elektrophysiologische und auch bildgebende Befunde. Kritische Differenzialdiagnosen stellen v. a. aufgrund ihrer Klinik und/oder Elektrophysiologie z.B. erregerbedingte (Borreliose, HIV, Diphtherie), hereditäre, amyloidotische oder paraproteinämische Polyneuropathien oder das POEMS-Syndrom (Polyneuropathie, Organomegalie, Endokrinopathie, MProtein, Hautläsionen/Skin) dar [5]. Darüber hinaus ist zu bedenken, dass bei Patienten mit Diabetes mellitus ein erhöhtes Risiko besteht, eine CIDP zu entwickeln, so dass ein rasche Verschlechterung einer diabetischen Polyneuropathie auch an eine zusätzliche CIDP denken lassen sollte. - Tab. 1 gibt einen Überblick über die klinischen Diagnosekriterien der European Federation of Neurological Societies/Peripheral Nerve Society Guideline [5].

\section{Merke}

Eine frühzeitige Diagnosestellung ist essenziell, um eine irreversible, mitunter sekundäre axonale Schädigung zu verhindern.

\section{Immunpathogenese}

Autoptische Befunde zeigen bei ca. der Hälfte aller CIDPPatienten demyelinisierende und axonale Schädigungszeichen in den Spinalwurzeln und den Spinalganglien sowie lymphozytäre Infiltrationen. Ein klares Antigen konnte bisher in den meisten Fällen nicht gefunden werden - additive humorale Faktoren, Komplementaktivierung und eine BZell-vermittelte Zusatzpathogenese werden vermutet. 
- Tab. 1 Klinische Diagnosekriterien der CIDP (European Federation of Neurological Societies/Peripheral Nerve Society Guidelines) [5].

\begin{tabular}{|c|c|c|}
\hline Einschlusskriterien & Ausschlusskriterien & Supportive Kriterien \\
\hline $\begin{array}{l}\text { - typische CIDP: } \\
\text { - chronisch progressiv oder schubartig } \\
\text { - symmetrische proximale und distale } \\
\text { Schwäche } \\
\text { - sensible Symptome aller Extremitäten } \\
\text { - Entwicklung }>2 \text { Monate } \\
\text { - fehlende oder abgeschwächte } \\
\text { Muskeleigenreflexe } \\
\text { - atypische CIDP: } \\
\text { - vorwiegend distal (DADS) } \\
\text { - oder asymmetrisch (MADSAM) } \\
\text { - oder fokal } \\
\text { - rein motorisch } \\
\text { - oder rein sensibel } \\
\text { - elektrophysiologische Kriterien** (mind. } 1 \text { der } \\
\text { folgenden Kriterien in mind. } 2 \text { Nerven): } \\
\text { 1. dmL } \geq 50 \% \text { über dem OG außer N. medianus } \\
\text { 2. F-Wellen-Latenz } \geq 30 \% \text { über OG oder feh- } \\
\text { lend (wenn MSAP-Ampl. } \geq 1 \text { mV) } \\
\text { 3. NLG-Reduktion } \geq 30 \% \text { des unteren } \\
\text { Grenzwerts } \\
\text { 4. temporale Dispersion ( } \geq 30 \% \text { verlängerte } \\
\text { MSAP-Dauer prox. vs. distal) } \\
\text { 5. distale MSAP-Dauer signifikant verlängert } \\
\text { 6. inkompletter Leitungsblock ( }>50 \% \text { Amp- } \\
\text { litudenreduktion prox. vs. distal) oder ein } \\
\text { weiteres demyelinisierendes Zeichen } 1-5 \text {, } \\
\text { wenn nur in } 1 \text { Nerv Leitungsblock }\end{array}$ & 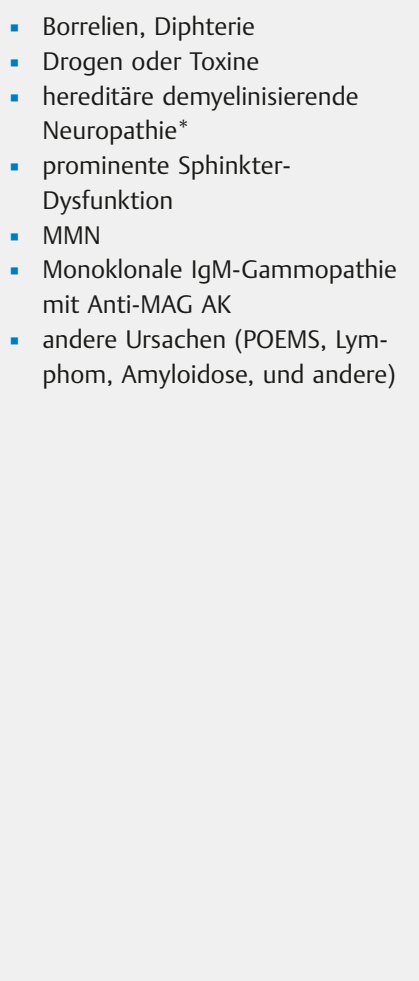 & $\begin{array}{l}\text { 1. erhöhtes Liquoreiweiß mit Zell- } \\
\text { zahl <10/ml } \\
\text { 2. MRT mit Gadolinium-Enhance- } \\
\text { ment und/oder Hypertrophie } \\
\text { der Cauda equina, der lumbosa- } \\
\text { kralen oder zervikalen Nerven- } \\
\text { wurzeln oder des Plexus barialis } \\
\text { oder Plexus lumboscralis } \\
\text { 3. abnorme sensible Elektrophy- } \\
\text { siologie in mind. } 1 \text { Nerv: } \\
\text { - } \text { normale Neurografie des N. } \\
\text { suralis mit abnormen Befun- } \\
\text { den im N. medianus (außer } \\
\text { Karpaltunnelsyndrom) oder } \\
\text { abnorme SNAP-Amplitude des } \\
\text { N. radialis oder } \\
\text { - NLG <80\% oder NLG <70\%, } \\
\text { wenn SNAP-Amplitude <80\% } \\
\text { oder } \\
\text { - verzögerte somatosensorisch } \\
\text { evozierte Potenziale (ohne } \\
\text { Vorliegen einer ZNS } \\
\text { Erkrankung) } \\
\text { 4. klinische Verbesserung nach } \\
\text { immunmodulatorischer } \\
\text { Therapie } \\
\text { 5. typische Befunde der } \\
\text { Nervenbiopsie }\end{array}$ \\
\hline \multicolumn{3}{|l|}{ Diagnosen } \\
\hline \multirow[t]{2}{*}{ definitive Diagnose } & $\begin{array}{l}1 \text { Kriterium von } 1-6 \text { erfüllt } \\
\text { oder }\end{array}$ & \\
\hline & wahrscheinliche Diagnose & plus 1 supportives Kriterium \\
\hline \multirow[t]{2}{*}{ wahrscheinliche Diagnose } & $\begin{array}{l}\text { möglicher LB ( } \geq 30 \% \text { Reduktion) in } 2 \\
\text { Nerven } \\
\text { oder }\end{array}$ & \\
\hline & mögliche Diagnose (siehe unten) & plus 1 supportives Kriterium \\
\hline mögliche Diagnose & $\begin{array}{l}1 \text { Kriterium von 1-6 in nur } 1 \text { Nerv } \\
\text { erfüllt }\end{array}$ & \\
\hline
\end{tabular}

Spezifische Triggerfaktoren werden kontrovers und uneinheitlich diskutiert.

Immunpathologisch finden sich Assoziationen der CIDP zu anderen Erkrankungen, z. B. Transplantationen (Graft-versus-Host-CIDP), Infektionen wie HIV, Zytomegalie (CMV), Campylobacter jejuni oder Hepatitis C, aber auch zu monoklonalen Gammopathien (paraproteinämische Neuropathien) sind Überlappungen beschrieben, denen wir einen eigenen Absatz widmen möchten. Eine erhöhte Inzidenz wird außerdem bei Patienten mit Diabetes mellitus und CMT (erbliche Charcot-Marie-Tooth-Neuropathie) vermutet [3].

\section{Elektrophysiologische Befunde}

Wichtig bei der Untersuchung peripherer Nerven bei V. a. immunvermittelte Pathogenese sind vor allem die Durchführung einer ausgedehnten Elektrophysiologie inkl. Messung von F-Wellen, proximalen Stimulationen (z. B. am Oberarm oder über Erb) und die Messung 
beider Seiten mit der Frage nach reduzierter Nervenleitgeschwindigkeit, verzögerter distal motorischer Latenz, verlängerter Dauer der Amplitude des distalen Muskelsummenaktionspotenzials (MSAP), temporaler Dispersion oder inkomplettem und komplettem Leitungsblock außerhalb physiologischer Engstellen. -Abb. 1 zeigt einen klassischen Befund einer demyelinisierenden Läsion des N. medianus.

Vorsicht

Bei langen und chronischen Verlaufsformen dominieren außerdem oft sekundäre axonale Schädigungszeichen und erschweren somit die Diagnostik nach vorgegebenen Kriterien.

Von den zahlreichen Leitlinien hat die neueste Version der European Federation of Neurological Societies/Peripheral Nerve Society Guideline [5] mit einer Sensitivität von $81 \%$ und einer Spezifität von $96 \%$ insgesamt eine gute Alltagstauglichkeit zeigen können. Diese Leitlinien nennen außerdem mehrere supportive Kriterien, die als hilfreich bzw. diagnoseunterstützend zu Rate gezogen werden können ( $\triangleright$ Tab. 1). Eine Aussparung des N. suralis, v.a. in der Frühphase der Erkrankung bei gleichzeitigen Auffälligkeiten in den SEP oder den SNAP anderer Nerven (sog. „sural sparing“), wird als typisch für eine CIDP angesehen. Es sollte nicht unerwähnt bleiben, dass dieses sural sparing auch häufig bei der akuten Polyneuritis, dem Guillain-Barré-Syndrom, gefunden wird.

\section{Labor}

Neben klassischen Standardlaboruntersuchungen (Leber-, Nierenwerte, B-Vitamine, Glukose, TSH) sollte bei einem klassisch demyelinisierenden Muster unbedingt die Diagnostik um eine Serumelektrophorese mit Immunfixation (zur Abgrenzung gegen eine paraproteinämische Neuropathie oder ein POEMS) und eine HIV-Testung ergänzt werden. In seltenen Fällen, v. a. bei jüngeren Patienten, kann die Bestimmung langkettiger Fettsäuren, von Arylsulfatase A oder bestimmter Enzyme zum Ausschluss von Lipidspeichererkrankungen nötig sein, wobei hier meist zusätzliche zentrale Symptome dominieren.

Die Bestimmung möglicher Anti-Gangliosid- und Sulfatid-Antikörper hingegen spielt eine untergeordnete Rolle. Im Liquor findet sich häufig eine zytalbuminäre Dissoziation (d.h. eine deutliche Liquoreiweißerhöhung $>45 \mathrm{mg} / \mathrm{dl}$ bei fehlender bis nur geringer Zellzahlerhöhung $<10 / \mathrm{mm}^{3}$ ). Hier ist jedoch sowohl Spezifität als auch Sensitivität eingeschränkt, Häufigkeitsangaben schwanken je Verteilung von 34\% (bei MADSAM) bis zu $95 \%$ (bei klassischer CIDP).

\section{Nervenbiopsie}

Die Nervenbiopsie, vorzugsweise des $\mathrm{N}$. suralis, bringt häufig die richtige Diagnose zu Tage (siehe Immunpathogenese), kann jedoch auch falsch negativ sein. Dies beruht auf der Tatsache, dass man aufgrund der Multifokalität der entzündlichen Veränderungen mitunter den Ort der Entzündung verpassen kann. Hier könnte nach unserer Ansicht eine Kombination mit Befunden der Bildgebung (z. B. deutliches Ödem im Ultraschall, T2-Läsion oder KM-Enhancement im MRT) und/oder der Elektrophysiologie in der Auswahl einer geeigneten Biopsiestelle hilfreich sein - dieser Ansatz müsste jedoch in einer prospektiven Kohorte analysiert werden.

Die Nervenbiopsie zeigt in seltenen Fällen bei der CIDP eine perivaskuläre, perineurale und epineurale Infiltration von TZellen, häufiger hingegen Infiltration von Makrophagen mit Invasion der Schwann-Zellmembran. Als Zeichen der De- und Remyelinisierung finden sich oft sog. Zwiebelschalenformationen („onion bulbs“, \Abb. 1).

\section{Bildgebung}

Sowohl MRT als auch Ultraschall zeigen in bis zu $90 \%$ aller Patienten mit CIDP multilokuläre oder generalisiert verdickte Nerven [6, 7]. Sonografisch findet sich dies vor allem an ( Abb. 1):

- zervikalen Wurzeln

- Plexus brachialis

- Oberarmnerven

Die Echogenität der Nerven kann erhöht oder reduziert sein. Wahrscheinlich reagieren Patienten mit hypoechogenen Nerven (als Zeichen einer akuten Inflammation) nach unserer Erfahrung besser auf eine Therapie. Hyperechogene Veränderungen scheinen Zeichen der Chronifizierung i.S. von perifaszikulärer Fibrose zu sein, doch weitere Erfahrungen und Studien sind hier notwendig. In seltenen Fällen finden sich auch bildmorphologisch gänzlich unauffällige Nerven.

Im MRT kann zusätzlich zum Ultraschall der Plexus lumbosacralis sowie die Kaudafasern dargestellt sowie (meist nur moderate) Kontrastmittelaufnahmen als Entzündungsäquivalent gezeigt werden. Die Verteilung der Auffälligkeiten ist zumeist symmetrisch (mit Ausnahme der MADSAM) im Gegensatz zur MMN, bei denen Nervenschwellungen häufig asymmetrisch vorkommen.

Merke

Die CIDP ist die häufigste chronische immunvermittelte Neuropathie. Neben multifokalen Zeichen der Demyelinisierung in der Elektrophysiologie findet sich meist eine zytalbuminäre Dissoziation im Liquor. Die Bildgebung peripherer Nerven und Wurzeln kann zur Diagnosefindung beitragen.

\section{Therapie}

Die Therapie der CIDP hat sich in den letzten Jahren nur unwesentlich geändert. Klasse-I-Evidenz besteht immer noch nur für die Durchführung regelmäßiger Immunglobulininfusionen mit variabler Gesamtdosis und 
Intervalldauer, regelmäßiger Steroidgaben (p.o. oder auch als regelmäßige Stoßtherapie) und Plasmaaustauschverfahren. Die subkutane Immunglobulintherapie scheint eine weitere Option zur Therapie darzustellen und ist besonders bei Patienten, die unabhängig bleiben müssen (z.B. Berufstätige, weit von Zentren entfernt Lebende) eine Zukunftsoption.

Für genauere Dosierungen empfehlen wir die Leitlinien der DGN. Additive Therapien, z. B. Azathioprin, Mycophenolatmofetil, Cyclosporin A, Cyclophosphamid oder Rituximab, konnten bisher keine Evidenzklasse I erlangen. Wir raten allerdings, individuell derartige Therapieschritte je nach klinischem Schweregrad, Verlauf und Basistherapiebedarf unter Beachtung der üblichen Kautelen zu evaluieren. Experimentelle Ansätze mittels Interferon beta, Fingolimod, Natalizumab, Bortezomib oder Eculizumab (Medikamente, die teilweise aus der Behandlung der Multiplen Sklerose bekannt sind) blieben bisher oftmals den positiven Nutzenbeweis schuldig.

\section{Paranodopathien}

In den letzten Jahren wurden vermehrt Fälle von immunvermittelten Neuropathien veröffentlicht, bei denen IgG4Antikörper gegen paranodale Strukturen entdeckt wurden $[8,9]$. Hierbei wurden bisher Caspr1, Contactin1- und Neurofascin-155-Antikörper beschrieben. Diese Gruppe ist mit $<10 \%$ der CIDP-Formen zwar selten, jedoch therapeutisch relevant, da hier insbesondere ein schlechtes Ansprechen auf Immunglobuline beschrieben ist.

Im Gegensatz zur klassischen CIDP werden histologisch keine makrophagenvermittelten Demyelinisierungen oder Zwiebelschalen entdeckt, sondern Schädigungen der Ranvier-Schnürringe mit deutlicher axonaler Beteiligung (Paranodien sind bei der klassischen CIDP meist ausgespart). Elektrophysiologisch hingegen zeigt sich ein „klassisches“ Bild mit Leitungsblocks und F-WellenVerzögerungen. Klinisch bieten diese Patienten oft einen schnelleren Verlauf mit z.T. schweren motorischen Symptomen, sensibler Ataxie und Aktionstremor.

\section{Merke}

All diesen Fällen war ein eher schlechtes Ansprechen auf Standardtherapien (Immunglobuline, Steroide oder Plasmaaustausch) gemeinsam. Diese Patienten sollten bereits frühzeitig einer immunmodulatorischen AntiCD-20-Antikörpertherapie, z. B. mit Rituximab, zugeführt werden.

Zukünftige Projekte werden zeigen, inwieweit hier eine CIDP-Subgruppe vorliegt oder ob langfristig eigene Begriffsdefinitionen zur Unterscheidung nötig werden. Bereits jetzt sollte man als Therapeut jedoch bei Fällen einer bisherigen CIDP mit mangelhaftem Therapieansprechen daran denken, dass es sich in diesen Fällen um eine Paranodopathie handeln könnte, und eine weitergehende Diagnostik, z. B. durch ein wissenschaftlich engagiertes Zentrum, durchführen lassen.

\section{FALLBEISPIEL 2}

Ein 67-jähriger Patient beklagt seit 8 Jahren Dysästhesien der Füße, intermittierend auch der Hände mit z. T. sehr unangenehm stechenden Schmerzen. Das Laufen sei hierdurch erschwert. Er sei sonst gesund. Vor Jahren sei eine demyelinisierende Polyneuropathie festgestellt worden. Mehrere Versuche mit Steroiden und Immunglobulinen hätten keinen Effekt gehabt.

In der hiesigen Untersuchung bestätigt sich die demyelinisierende Polyneuropathie, jedoch mit auffallenden Verzögerungen der distal motorischen Latenz in allen Nerven. In der ergänzten Laboruntersuchung findet sich eine monoklonale Gammopathie Typ IgM. Aufgrund der elektrophysiologischen Befunde ergänzten wir die Anti-MAG-Antikörper, die hochpositiv waren. Daraufhin wurde eine Therapie mit Rituximab eingeleitet. Es handelte sich um eine paraproteinämische Neuropathie mit Anti-MAG-IgM Antikörpern.

\section{Paraproteinämische Neuropathien}

\section{Klinische Präsentation}

Die paraproteinämischen Neuropathien bieten ein buntes Potpourri aus verschiedenen mit einer monoklonalen Gammopathie unklarer Signifikanz (MGUS), einem multiplen Myelom oder Morbus Waldenström assoziierten Neuropathien $[10,11]$.

Zum einen gibt es IgA-, IgG- und IgM-assoziierte demyelinisierende Verlaufsformen, die man als MGUS-CIDP bezeichnet. Deren Verlauf ist relativ identisch zur klassischen CIDP. Darüber hinaus existiert die oft durch distal symmetrische und sensible Symptome dominierende Verlaufsform (DADS), welche v.a. bei IgM-Gammopathien vorkommt.

Zum anderen gibt es die überwiegend axonalen Formen, die sowohl beim multiplen Myelom als auch beim MGUS vorkommen und deren klinische Präsentation oft aus überwiegend sensiblen und sensoalgetischen Symptomen besteht. Hier ist eine immunvermittelte Genese nicht bestätigt und die Behandlung der hämatologischen Erkrankung steht im Vordergrund.

Zusätzlich gibt es die mit multiplem Myelom, Organvergrößerungen, Endokrinopathie und Hautläsionen einhergehende POEMS-Variante, eine oft schmerzhafte, sensomotorische demyelinisierende Polyneuropathie. 
Neben klassischen Myelommanifestationen finden sich hier häufig Auffälligkeiten der Haut (inkl. Ödemen, Hautverfärbungen, Nävi) und erhöhte Serum-Titer des vascular endothelial growth factor (VEGF). Der Verlauf ist oft schwer und rasch. Eine Abgrenzung zu einer MGUS-CIDP ist oft schwierig [1].

Zuletzt wird die in ca. $50 \%$ der Fälle einer IgM-MGUS vorkommende DADS-Variante mit Anti-MAG-IgM-Antikörpern beschrieben (myelinassoziiertes Glykopeptid, MAG). Hier zeigt sich neben distal betonten sensiblen Symptomen vor allem eine sensorische Ataxie, oft manifest durch einen ausgeprägten Extremitätentremor. Die Klinik ist meistens chronisch progredient.

\section{Immunpathogenese}

Den Anti-MAG-Antikörpern wird eine immunmediierende Eigenschaft zugeordnet, mit Aktivierung von Komplement, Ablagerung von M-Proteinen und Myelinschädigung durch Aufweitung der Myelinlamellen im Bereich der Schwann-Zellen, aber auch in paranodalen Schleifen und im Bereich der Schmidt-Lantermann-Inzisuren. Beim POEMS findet man T-Zell-Infiltrate in den Spinalwurzeln, endoneuronale Ödeme und vaskuläre Veränderungen (VEGF-assoziiert), jedoch keine Makrophagen [10].

Merke

Paraproteinämische Neuropathien sind häufig. Das gemeinsame Auftreten von Polyneuropathie und Gammopathie sollte stets an eine immunvermittelte Genese denken lassen, deren Klinik und Elektrophysiologie oft den Befunden bei CIDP ähnelt.

\section{Elektrophysiologie}

Die MGUS-CIDP präsentiert sich elektrophysiologisch wie eine klassische CIDP, daneben existiert eine Variante mit deutlicher Betonung/Verlängerung der distal motorischen Latenzen (DADS). Die der multiplen Myelom oder der MGUS-assoziierten Polyneuropathie ist überwiegend axonal, die POEMS-Variante zeigt uniforme Demyelinisierungen und kann dadurch u. U. von einer MGUS-CIDP unterschieden werden. Letztlich zeigt die Anti-MAG-Neuropathie meist den DADS-Typ mit deutlich verlängerten distal motorischen Latenzen.

\section{Weitere Zusatzuntersuchungen}

Die Diagnose ergibt sich oftmals aus der Serologie (ProteinElektrophorese mit Nachweis eines Extragradienten) oder der Urindiagnostik. Die meisten Patienten mit immunvermittelter paraproteinämischer PNP zeigen eine Eiweißerhöhung im Liquor, vergleichbar der klassischen CIDP.

Die Rolle der Bildgebung bei paraproteinämischen Neuropathien ist noch weitestgehend unklar, wenngleich MGUSCIDP-, DADS- und Anti-MAG-Patienten ebenso multifokale Nervenschwellungen mit jedoch meist geringerem Ausmaß als die CIDP zeigen. Beschreibungen bei POEMS-
Patienten nennen bisher vor allem multiple Engpassnervenschwellungen als typisch, wenngleich hier unserer Ansicht nach valide Daten fehlen [6]. Bei den anderen axonalen Verlaufsformen gammopathieassoziierter Neuropathien hingegen finden sich keine sonografischen Besonderheiten.

\section{Vorsicht}

Man sollte stets bei zufällig festgestellter monoklonaler Gammopathie eine hämatologische Mitbeurteilung veranlassen.

\section{Therapie}

Das Fallbeispiel zeigt das Dilemma bei paraproteinämischen Neuropathien. Diejenigen Neuropathien, die ein CIDP-ähnliches Muster zeigen, reagieren meist gut auf die gleichen Therapien wie die klassische Form. Die IgMGammopathie, vor allem diejenige, die mit Anti-MAGAntikörpern assoziiert ist, scheint hingegen eine schlechte Response auf klassische Therapien zu zeigen. Dies führt häufig zu frustranen Therapieverläufen. Es gibt hingegen Hinweise auf eine gute Suszeptibilität für Rituximab, weswegen dieses Therapieverfahren bei dieser Verlaufsform als beste Option genannt wird.

\section{Merke}

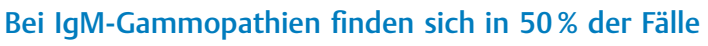
Anti-MAG-Antikörper. Patienten reagieren dann oft unzureichend auf Standardtherapien der CIDP.

FALLBEISPIEL 3

Ein 54-jähriger Mann präsentiert sich mit einer rasch progredienten atrophen Handparese links mit Faszikulationen und wird mit der Verdachtsdiagnose einer amyotrophen Lateralsklerose in ein neuromuskuläres Zentrum eingewiesen.

In der Untersuchung zeigen sich rein motorische Paresen im M. extensor digitorum communis (M3), im M. abductor pollicis brevis und im M. flexor pollicis longus (M4-), passend zum Versorgungsgebiet des N. medianus und N. radialis. Die Elektrophysiologie zeigt eine Minderung der MSAP-Amplitude im N. radialis und lediglich einen möglichen Leitungsblock im N. medianus ( $\mathbf{A}$ Abb. 2). Die SNAP sind regelrecht. Im Ultraschall der peripheren Nerven findet sich eine proximale Nervenschwellung des $\mathrm{N}$. radialis und $\mathrm{N}$. medianus im Vergleich zur unauffälligen Gegenseite ( Abb. 2).

Unter der Verdachtsdiagnose einer immunvermittelten Genese werden 0,4 g/kg KG Immunglobulin über 5 Tage verabreicht. Dies führt bereits innerhalb von 14 Tagen zu einer signifikanten Besserung (Vigorimetrie von 0,4 auf 0,8 mbar, M. extensor digitorum brevis M4+, Daumenmuskulatur M5). Im Nachgang finden sich in den vor der ersten IVIGGabe bestimmten Blutuntersuchungen positive IgM-GM1-Antikörper. 

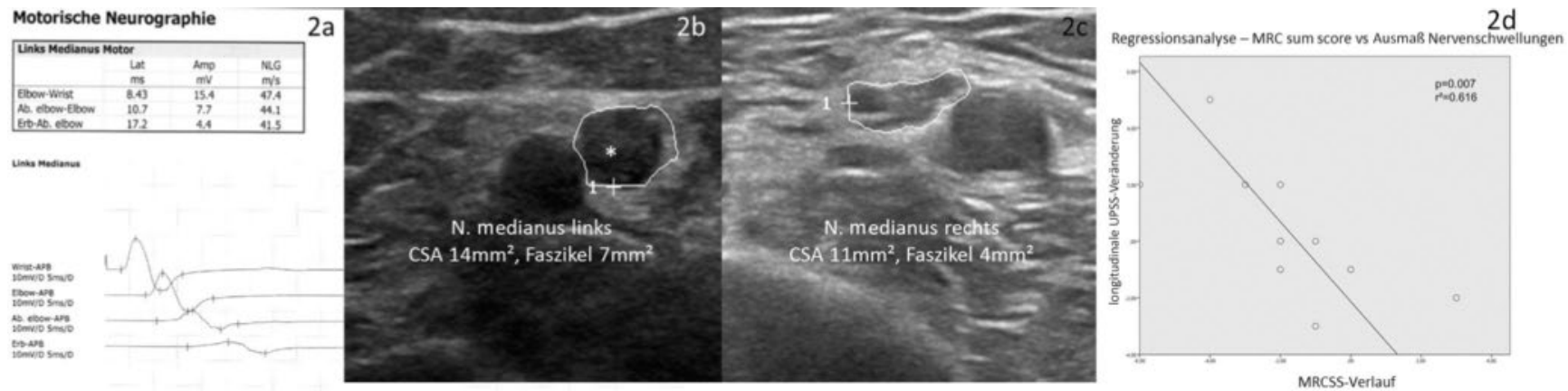

Abb. 2 Neurografie des linken N. medianus (Quelle: Rattay TW, Winter N, Décard B et al. Nerve ultrasound as follow-up tool in treated multifocal motor neuropathy. Eur J Neurol 2017; 24: 1125-1134 [13]).

a CMAP-Amplitudenreduktion $>50 \%$ von distal nach proximal ohne signifikante Flächenzunahme, passend zu einer progredienten Amplitudenminderung mit möglichem Leitungsblock sowie im Verlauf temporale Dispersion; b Interessanterweise findet sich am Oberarm desselben Nervs eine signifikante, hypoechogene Faszikelschwellung ( $\left.{ }^{*}\right)$; $\mathbf{c}$ die am nicht betroffenen Arm nicht zu sehen ist; $\mathbf{d}$ Verlauf von Nervenschwellungen unter Therapie in Korrelation zur Klinik bei insgesamt 12 Patienten mit multifokaler motorischer Neuropathie.

\section{Multifokale motorische Neuropathie (MMN)}

\section{Klinische Präsentation}

Asymmetrische, rein motorische Paresen im Versorgungsgebiet zweier oder mehrerer Nerven, oftmals distale Armmuskeln betreffend, sind das Kernsymptom der MMN. Zeichen einer Läsion des 1. Motoneurons sind nicht vorhanden. Die Reflexe in betroffenen Segmenten sind oft - jedoch nicht immer - abgeschwächt. Die Muskeln sind oft atroph, z.T. auch faszikulierend. Patienten beklagen häufig Krämpfe und Erschöpfungszustände. Männer mittleren Alters sind häufiger betroffen. Das Fortschreiten der Erkrankung ist chronisch progredient oder stufenweise. In seltenen Fällen können Hirnnerven betroffen sein, sensible Nerven sind i.d.R. nicht betroffen, wenngleich sensible Symptome in jedem 5 . Fall geschildert werden [2, 12].

\section{Immunpathogenese}

In $50-70 \%$ der Patienten finden sich IgM-Antikörper gegen Gangliosid GM1, wenngleich diese Antikörper mitnichten pathognomonisch für die Erkrankung sind und zumindest niedrigtitrig - auch bei der amyotrophen Lateralsklerose vorkommen können [1]. Es kommt durch Komplement- und Makrophagenaktivierung zu einer Schädigung von Myelin und Axon, vereinzelt auch zur Bildung von Zwiebelschalenphänomenen analog der CIDP.

\section{Elektrophysiologische Befunde}

In der Untersuchung der peripheren Nerven liegt das Hauptaugenmerk für die Diagnosestellung auf 2 Kernpunkten:

- Suche nach Leitungsblocks außerhalb von allgemeinen Engpässen

- Aussparung sensibler Nerven
Letzteres kann bei konkurrierenden Diagnosen, die eine Polneuropathie auslösen können (z. B. stattgehabte Chemotherapie, Diabetes mellitus, Vitamin-B12-Mangel), fehlen. Ersteres bereitet vor allem bei sehr distalen oder aber sehr proximalen Leitungsblöcken Schwierigkeiten. Insgesamt liegt die Trefferquote bei der MMN auch bei ausführlicher Suche nur bei $80 \%[12,13]$.

\section{Labor}

Laboruntersuchungen, v. a. die Suche nach den im Fallbeispiel erwähnten GM1-IgM-Antikörpern, können in vielen Fällen hilfreich sein (ca. 70\%). Liquordiagnostische Untersuchungen zeigen zwar in ca. 30\% eine Eiweißerhöhung, sind jedoch nicht diagnoseunterstützend.

\section{Bildgebung}

In den letzten Jahren hat sich die Bildgebung der peripheren Nerven als sehr hilfreich erwiesen. Sowohl im MRT als auch im hochauflösenden Ultraschall finden sich in mehr als $75 \%$ der Fälle multifokale, meist asymmetrische Nervenschwellungen bzw. T2-Hyprintensitäten, oft in den proximalen Nervenabschnitten (auch bei distalen Paresen), evtl. mit mäßigen KontrastmittelEnhancement, die somit den entzündlichen Charakter motorischer Ausfälle untermauern [6]. Nervenschwellungen und elektrophysiologischer Leitungsblock finden sich oft nicht an den gleichen Stellen [13]. Diese Befunde erleichtern eine Unterscheidung von anderen rein motorischen Neuro- oder Motoneuronopathien [6]. Unter erfolgreicher Therapie sind die Schwellungen der Nerven, v.a. einzelne Faszikel betreffend, oft regredient [13] ( Abb. 2).

\section{Therapie}

Die Therapie bei der MMN beschränkt sich aktuell auf die regelmäßige i.v.-Applikation von Immunglobulinen 
(Leitlinien der DGN [14]). Die subkutane Immunglobulingabe scheint ebenso wirksam zu sein. Plasmaseparation ist nicht hilfreich, Steroide können die Symptome mitunter verschlechtern. Dennoch zeigt sich die Krankheit bei vielen Patienten oftmals langsam progredient, wenngleich der Verlauf insgesamt signifikant verzögert werden kann. Andere Therapieverfahren, z. B. Cyclophosphamid, Azathioprin, Mycophenolatmofetil oder immunmodulierende Verfahren (Anti-CD-20-Antikörper), zeigen allenfalls geringen Erfolg.

\section{Merke}

Asymmetrische rein motorische atrophe Paresen können ein Hinweis auf eine MMN sein, v. a. wenn mehrere Nerven betroffen sind. Häufige Fehldiagnosen sind eine Motoneuronerkrankung oder Engpass-Syndrome. Leitungsblocks sind bei der Diagnosestellung hilfreich, jedoch nicht obligat.

\section{Vaskulitische Polyneuropathie}

\section{Klinische Präsentation}

Eine weitere Ursache für eine entzündlich bedingte Polyneuropathie ist eine Entzündung der Vasa nervosum. Hierbei unterscheidet man eine isolierte Vaskultis des peripheren Nervensystems (nonsystemic vasculitic neuropathy, NSVN) von einer Polyneuropathie im Rahmen einer systemischen Erkrankung.

Der Verlauf kann schubweise auftreten, aber auch in bis zu $30 \%$ der Fälle einen chronischen Prozess aufweisen $[15,16]$. Die typische Klinik einer Mononeuritis multiplex, in der einzelne Nervenausfälle über die Zeit auftreten, ist aber nur in der Minderheit der Fälle zu finden. Viel häufiger finden sich Schwerpunktneuropathien oder gar symmetrische, distal betonte Polyneuropathien. Ein besonderes klinisches Charakteristikum ist der neuropathische Schmerz, der oft auch plötzlich im Falle einer Nervenischämie auftreten kann. Insbesondere aufgrund der häufig fluktuierenden, variablen klinischen Präsentation ist die Diagnose anspruchsvoll - die mittlere Dauer bis zur Diagnose beträgt bei der nicht systemischen vaskulitischen Neuropathie etwa 5-8 Monate.

\section{Merke}

Die Minderheit der Patienten mit einer vaskulitischen Polyneuropathie hat eine PNP vom Multiplex-Typ, vielmehr ein generalisiertes Muster mit Schwerpunkten und Asymmetrien. Schmerz ist immer vorhanden.

\section{Immunpathogenese}

Die Entzündung der nervenversorgenden Gefäße ist der gemeinsame pathophysiologische Mechanismus. Die Entzündung der Vasa nervosum führt schließlich zu Thrombose, Gefäßverschluss und Ischämie des Nerven [17]. Allerdings kann die Ätiologie der Vaskulitis unterschiedlich sein. $>$ Tab. 2 zeigt die Einteilung der vaskulitische Polyneuropathien [15, 16, 17].

\section{Elektrophysiologische Befunde}

Elektrophysiologische Befunde zeigen insbesondere Zeichen eines axonalen Verlusts von motorischen und sensiblen Nervenfasern an. Das bedeutet insbesondere eine Reduktion der Amplituden der motorischen und sensiblen Summenpotenziale in der Neurografie [18]. Die EMG zeigt entsprechend Zeichen akuter (positive scharfe Wellen und Fibrillationen) und chronischer Denervierung (mit einer Amplitudenvergrößerung der Potenziale motorischer Einheiten). Kurz nach Symptombeginn kann auch ein transienter Leitungsblock in der Neurografie auffallen, wenn sich distal des Nerveninfarkts in der Frühphase noch keine Waller-Degeneration entwickelt hat [15].

\section{Ultraschall}

Der Durchmesser der peripheren Nerven ist im Vergleich zu Gesunden bei der vaskulitischen Polyneuropathie moderat erhöht [19], aber nicht so ausgeprägt wie bei chronisch demyelinisierenden Polyneuropathien. Insgesamt sind aber das Muster einer axonalen Polyneuropathie in der Elektrophysiologie und leicht verdickte Nerven im Ultraschall sehr verdächtig auf eine Vaskulitis.

\section{Nervenbiopsie}

Die Nervenbiospie kann die Diagnose der Vaskulitis sichern. Während bei der systemischen Vaskulitis die Diagnose auch aus der Beteiligung anderer Organsysteme gestellt werden kann, ist die Nervenbiopsie bei der nicht systemischen vaskulitischen Neuropathie der Goldstandard zur definitiven Sicherung der Diagnose $[13,15]$. Dabei sollte ein Nerv biopsiert werden, der klinisch und elektroneurografisch betroffen ist. Die Nervenbiopsie zeigt bei der Vaskulitis insbesondere entzündliche Infiltrate in den Gefäßwänden bzw. die Folgen des Nerveninfarkts.

Merke In der Regel finden sich akute und chronische Veränderungen nebeneinander.

\section{Erweiterte Diagnostik}

Die Zuordnung einer Vaskulitis zu einer Ätiologie erfordert entsprechend auch eine breite differenzialdiagnostische Abklärung. Hierbei können zunächst spezielle Laboruntersuchungen hilfreich sein $>$ Tab. 3 [17, 18, 20]. Zusätzlich empfiehlt sich - besonders zur Abgrenzung zu systemischen Vaskulitiden - eine erweiterte Organdiagnostik (CT-Thorax-Abdomen, PET etc.).

\section{Therapie}

Gute Studien mit dem Endpunkt der Besserung der Polyneuropathie liegen leider nicht vor. Die Therapie fußt auf einer Immunsuppression, wobei die DGN-Leitlinien [14] 
- Tab. 2 Einteilung der vaskulitischen Polyneuropathien nach Ätiologie.

\begin{tabular}{|c|c|c|c|}
\hline Einteilung & Ätiologie & & \\
\hline \multirow{4}{*}{$\begin{array}{l}\text { Primär systemische } \\
\text { Vaskulitis }\end{array}$} & Gefäßgröße & granulomatös & nicht granulomatös \\
\hline & groß & $\begin{array}{l}\text { Riesenzellarteriitiden } \\
\text { Arteriitis cranialis } \\
\text { Takayasu-Arteriitis }\end{array}$ & \\
\hline & mittel & & $\begin{array}{l}\text { Polyarteriitis nodosa } \\
\text { Kawasaki-Erkrankung }\end{array}$ \\
\hline & klein & $\begin{array}{l}\text { Wegener-Granulomatose } \\
\text { Churg-Strauss-Syndrom }\end{array}$ & $\begin{array}{l}\text { mikroskopische Polyangiitis } \\
\text { kryoglobulinämische Vaskulitis } \\
\text { Behçet-Syndrom }\end{array}$ \\
\hline \multirow[t]{5}{*}{$\begin{array}{l}\text { Sekundär systemische } \\
\text { Vaskulitis }\end{array}$} & Kollagenosen & \multicolumn{2}{|l|}{$\begin{array}{l}\text { rheumatoide Arthritis } \\
\text { Lupus erythematodes } \\
\text { Sjögren-Syndrom } \\
\text { Sklerodermie }\end{array}$} \\
\hline & maligne Erkrankungen & \multicolumn{2}{|c|}{$\begin{array}{l}\text { lympho- und myeloproliferative Erkrankungen } \\
\text { Karzinome }\end{array}$} \\
\hline & $\begin{array}{l}\text { andere entzündliche } \\
\text { Grunderkrankungen }\end{array}$ & \multicolumn{2}{|l|}{$\begin{array}{l}\text { Morbus Crohn } \\
\text { Colitis ulcerosa } \\
\text { Sarkoidose }\end{array}$} \\
\hline & medikamenteninduziert & \multicolumn{2}{|c|}{$\begin{array}{l}\text { Hydralazin, Phenytoin, Thyreostatika, Thiazide, Penizillin, Sulfonamide, } \\
\text { Morphin, Kokain, Amphetamin }\end{array}$} \\
\hline & erregerbedingt & \multicolumn{2}{|c|}{ HBV, HCV, HIV, CMV, Lepra, Borrelien, HTLV-I } \\
\hline \multicolumn{4}{|l|}{$\begin{array}{l}\text { nicht systemische } \\
\text { Vaskulitis }\end{array}$} \\
\hline \multicolumn{4}{|l|}{$\begin{array}{l}\text { nicht systemische vaskuli- } \\
\text { tische Polyneuropathie }\end{array}$} \\
\hline \multicolumn{4}{|l|}{ neuralgische Amyotrophie } \\
\hline \multicolumn{4}{|l|}{$\begin{array}{l}\text { diabetische Radikuloplexo- } \\
\text { pathie (schmerzhaft und } \\
\text { nicht schmerzhaft) }\end{array}$} \\
\hline $\begin{array}{l}\text { nicht systemische Haut/ } \\
\text { Nerv-Vaskulitis }\end{array}$ & & & \\
\hline
\end{tabular}

Tab. 3 Laboruntersuchungen bei V.a. Vaskulitis [17, $18,20]$.

\begin{tabular}{|c|c|}
\hline Routine & V.a. Vaskulitis \\
\hline $\begin{array}{l}\text { Blutbild } \\
\text { Elektrolyte } \\
\text { Leber- und } \\
\text { Nierenwerte } \\
\text { Urin } \\
\text { HbA1c, Glukose } \\
\text { BSG, CRP } \\
\text { Immunfixation } \\
\text { Komplement } \\
\text { Kryoglobuline } \\
\text { Hepatitis B, Hepatitis C }\end{array}$ & $\begin{array}{l}\text { antineutrophile zytoplasmische } \\
\text { Autoantikörper (cANCA, pANCA, } \\
\text { Anti-PR3, Anti-MPO) } \\
\text { ANA } \\
\text { ENA (SSA, SSB, Sm, RNP, Scl-70) } \\
\text { Rheumafaktoren } \\
\text { Anti-CCP-Antikörper } \\
\text { Liquor } \\
\text { HIV, Borrelien-AK, CMV } \\
\text { paraneoplastische AK (Anti-Hu) } \\
\text { ACE, löslicher Interleukin-2- } \\
\text { Rezeptor }\end{array}$ \\
\hline
\end{tabular}

hier Glukokortikoide und Cyclophsphamid (Grad III) empfehlen. Dabei soll ein Prednisolon-Äquivalent von 1 $\mathrm{mg} / \mathrm{kg} \mathrm{KG/d}$ oder eine Pulstherapie mit 500-1000 mg/d an 3-5 Tagen und anschließender Reduktion auf orale Erhaltungsdosis verbreicht werden. Für Cyclophosphamid empfiehlt sich eine Pulstherapie mit einer Induktion mit $350 \mathrm{mg} / \mathrm{m}^{2}$ KOF an 3 aufeinander folgenden Tagen und dann $600 \mathrm{mg} / \mathrm{m}^{2} \mathrm{KOF}$ in Abständen von 6-8 Wochen.

\section{Fazit}

Die Erkennung entzündlich vermittelter Neuropathien ist eine der fundamentalen Aufgaben in der Diagnostik der Polyneuropathie. Dies ist insbesondere deshalb essenziell, weil der Verlauf meist chronisch progredient ist und zu schweren neurologischen Behinderungen führt. 
Tab. 4 Klinische Präsentation und Zusatzbefunde bei immunvermittelten Neuropathien.

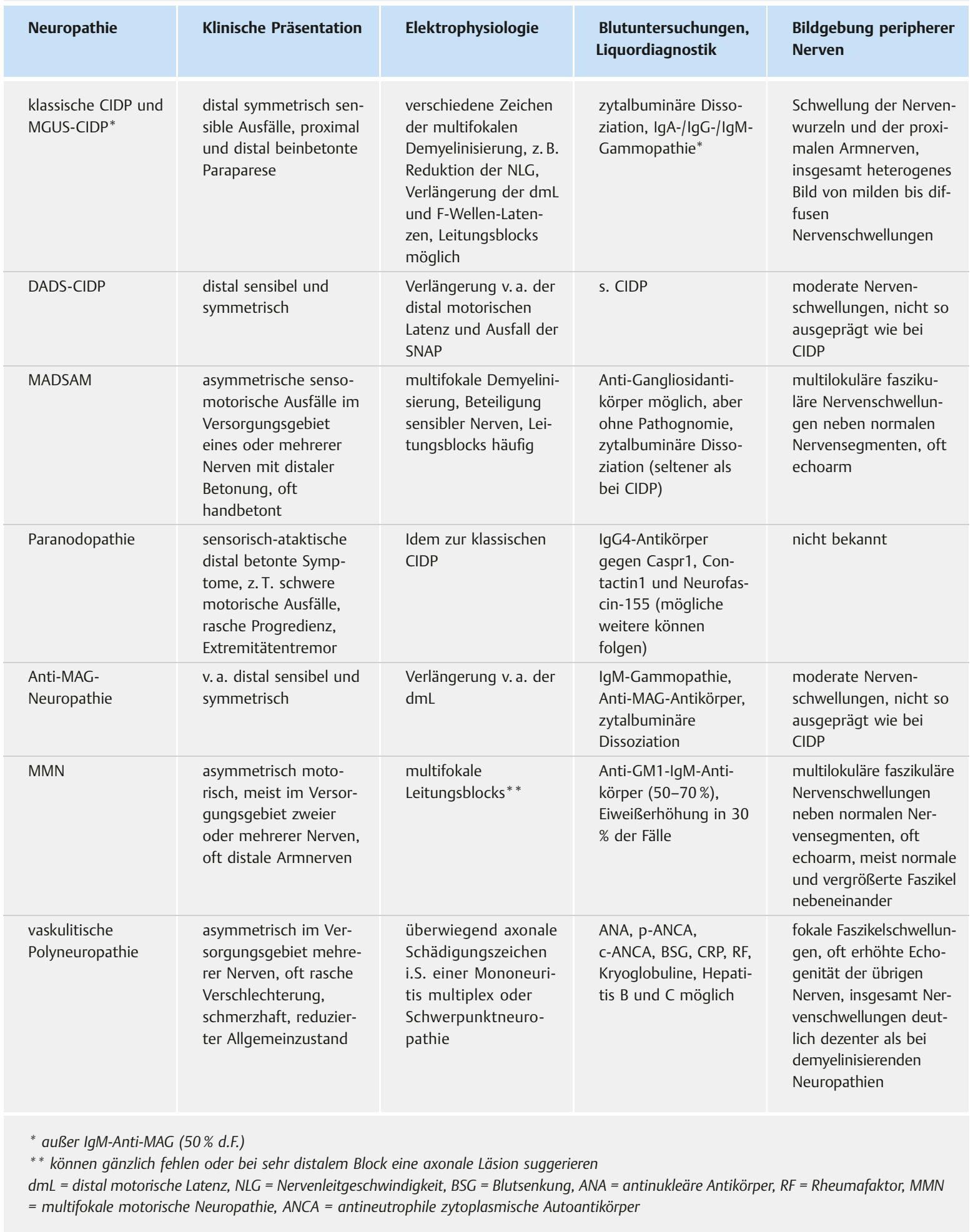

Die korrekte Einordung der entzündlichen Ursache der Neuropathie ist damit die Grundlage einer antiinflammatorischen Therapie.

Das Spektrum ist jedoch vielgestaltig, sowohl in klinischer als auch elektrophysiologischer und histopatho- logischer Ausprägung. Dabei sind i.d. R. Einzelbefunde für eine Diagnose nicht ausreichend. Es geht vielmehr um das Erkennen eines für eine Erkrankung spezifischen Musters ( $\triangleright$ Tab. 4) Die bildmorphologische Darstellung und Musteranalyse kann hier in Zukunft zusätzliche hilfreiche Informationen liefern. 
Therapeutisch stehen vor allem antiinflammatorische und entzündungsmodulierende Verfahren zur Verfügung. Weiterhin von hoher Relevanz ist die Suche nach Biomarkern, die eine gezielte Therapie erleichtern und auch den Verlauf wiedergeben.

\section{KERNAUSSAGEN}

- Inflammatorische Polyneuropathien führen unbehandelt zu signifikanten neurologischen Ausfällen.

- Die häufigste Form ist die chronisch inflammatorische demyelinisierende Polyradikuloneuropathie (CIDP).

- Des Weiteren finden sich mit überwiegend demyelinisierendem Schädigungsmuster die rein motorische multifokale motorische Neuropathie (MMN), paraproteinämische Neuropathien u. a.

- Die Paranodopathien zeigen eine eher axonale Schädigung bei demyelinisierender Elektrophysiologie.

- Die vaskulitische Polyneuropathie zeigt ein axonales Schädigungsmuster, hier muss eine weitere Einschätzung der Ätiologie der Vaskulitis erfolgen.

Eine frühe Diagnose ist essenziell, um eine antiinflammatorische Therapie einleiten zu können.

- Je nach Diagnose stehen als Therapie Glukokortikoide, Immunglobuline oder Plasmapherese zur Verfügung.

\section{Interessenkonflikt}

Die Autoren geben an, dass kein Interessenkonflikt vorliegt.

\section{Wissenschaftlich verantwortlich gemäß Zertifizierungsbestimmungen}

Wissenschaftlich verantwortlich gemäß Zertifizierungsbestimmungen für diesen Beitrag ist PD Dr. med. Alexander Grimm, Tübingen.

\section{Danksagung}

Dank an Frau Professor Dr. A. Bornemann, Institut für Neuropathologie, Universitätsklinikum Tübingen, für die Bereitstellung des histologischen Befundes in Fall 1.

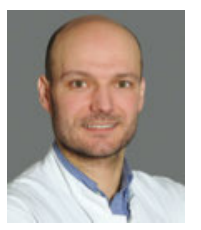

\section{Alexander Grimm}

PD, Dr. med. habil.; Studium der Humanmedizin an der Julius-Maximilians Universität Würzburg, 1999-2005, 2006 Promotion. 20062011 Facharztausbildung Neurologie an der Universitätsklinik Würzburg und Jena, 2011 Facharzt für Neurologie, seit 2013 Oberarzt für Neurologie, bis 2015 am Universitätsspital Basel, seit 2015 am Universitätsklinikum Tübingen, 2016 Habilitation; Leiter der klinischen Neurophysiologie, der Neurologischen Poliklinik sowie des neuromuskulären Zentrums.

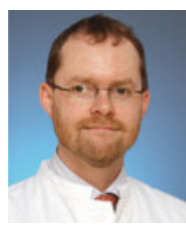

\section{Hubertus Axer}

apl. Prof. Dr. med. habil.; Studium der Humanmedizin an der RWTH Aachen, 19961997 AiP, 1997 Promotion, 1997-2002 Facharztausbildung Anatomie, RWTH Aachen, 2002 Habilitation, 2002 Facharzt für Anatomie, 2002-2007 Facharztausbildung Neurologie, Universitätsklinikum Jena; 2007 Facharzt für Neurologie; seit 2008 Oberarzt, 2011 Ernennung zum apl. Professor an der FSU Jena; Leiter der Klinischen Neurophysiologie und des Schwindelzentrums.

\section{Korrespondenzadresse}

PD Dr. med. Alexander Grimm

Neurologische Universitätsklinik Tübingen

Hoppe-Seyler-Str. 3

72072 Tübingen

E-mail: alexander.grimm@med.uni-tuebingen.de

\section{Literatur}

[1] Latov N. Diagnosis and treatment of chronic acquired demyelinating polyneuropathies. Nat Rev Neurol 2014; 10: 435-446

[2] Dalakas MC. Advances in the diagnosis, pathogenesis and treatment of CIDP. Nat Rev Neurol 2011; 7: 507-517

[3] Lewis RA. Chronic inflammatory demyelinating polyneuropathy. Curr Opin Neurol 2017; 30: 508-512

[4] Sinnreich M, Klein C], Daube JR et al. Chronic immune sensory polyradiculopathy: a possibly treatable sensory ataxia. Neurology 2004; 63: 1662-1669

[5] EFNS-PNS Task Force. European Federation of Neurological Societies/Peripheral Nerve Society Guideline on management of chronic inflammatory demyelinating polyradiculoneuropathy. Report of a joint task force of the European Federation of Neurological Societies and the Peripheral Nerve Society First Revision. Eur J Neurol 2010; 17: 356-363

[6] Gasparotti R, Padua L, Briani $C$ et al. New technologies for the assessment of neuropathies. Nat Rev Neurol 2017; 13: 203-216 
[7] Grimm A, Vittore D, Schubert $V$ et al. Ultrasound aspects in therapy-I CIDP compared to long-term treated CIDP. J Neurol 2016; 263: 1074-1082

[8] Querol L, Devaux J, Rojas-Garcia R et al. Autoantibodies in chronic inflammatory neuropathies: diagnostic and therapeutic implications. Nat Rev Neurol 2017; 13: 533-547

[9] Mathey EK, Garg N, Park SB et al. Autoantibody responses to nodal and paranodal antigens in chronic inflammatory neuropathies. J Neuroimmunol 2017; 309: 41-46

[10] Mauermann ML. Paraproteinemic neuropathies. Continuum (Minneap Minn) 2014; 20: 1307-1322

[11] Steck A], Stalder AK, Renaud S. Anti-myelin-associated glycoprotein neuropathy. Curr Opin Neurol 2006; 19: 458-463

[12] Nobile-Orazio E, Gallia F. Multifocal motor neuropathy: current therapies and novel strategies. Drugs 2013; 73: 397-406

[13] Rattay TW, Winter N, Décard B et al. Nerve ultrasound as follow-up tool in treated multifocal motor neuropathy. Eur J Neurol 2017; 24: 1125-1134

[14] Diener H-C. Leitlinien für Diagnostik und Therapie in der Neurologie. 5. Aufl. Stuttgart: Thieme; 2012

[15] Gwathmey KG, Burns TM, Collins MP et al. Vasculitic neuropathies. Lancet Neurol 2014; 13: 67-82
[16] Burns TM, Schaublin GA, Dyck PJB. Vasculitic neuropathies. Neurol Clin 2007; 25: 89-113

[17] Blaes F. Diagnosis and therapeutic options for peripheral vasculitic neuropathy. Ther Adv Musculoskelet Dis 2015; 7: 45-55

[18] Collins MP, Hadden RD. The nonsystemic vasculitic neuropathies. Nat Rev Neurol 2017; 13: 302-316

[19] Grimm A, Décard BF, Bischof A et al. Ultrasound of the peripheral nerves in systemic vasculitic neuropathies. J Neurol Sci 2014; 347: 44-44

[20] Collins MP, Dyck PJB, Gronseth GS et al. Peripheral Nerve Society: Peripheral Nerve Society Guideline on the classification, diagnosis, investigation, and immunosuppressive therapy of non-systemic vasculitic neuropathy: executive summary. J Peripher Nerv Syst 2010; 15: 176-184

\section{Bibliografie}

DOI https://doi.org/10.1055/s-0044-101831

Fortschr Neurol Psychiatr 2018; 86: 439-452

(c) Georg Thieme Verlag KG Stuttgart · New York ISSN 0720-4299 


\section{Punkte sammeln auf CM:thieme.de}

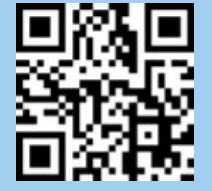

Diese Fortbildungseinheit ist 12 Monate online für die Teilnahme verfügbar.

Sollten Sie Fragen zur Online-Teilnahme haben, finden Sie unter http://cme.thieme.dehilfe

eine ausführliche Anleitung. Wir wünschen viel Erfolg beim Beantworten

der Fragen!

Unter eref.thieme.de/ZZYZ2CN oder über den QR-Code kommen Sie direkt

zum Artikel zur Eingabe der Antworten.

VNR 2760512018154651459

\section{Frage 1}

Welche der folgenden immunvermittelten Neuropathien imponiert i.d.R. nicht durch ein demyelinisierendes Muster in der Elektrophysiologie?

A Lewis-Sumner-Syndrom (MADSAM)

B multifokale motorische Neuropathie (MMN)

C vaskulitische Polyneuropathie

D POEMS

E chronisch inflammatorische demyelinisierende Polyradikuloneuropathie

\section{Frage 2}

Welche elektroneurografische Veränderung ist kein typisches Zeichen der Demyelinisierung?

A Reduktion der Nervenleitgeschwindigkeit

B Verlängerung der F-Wellen-Latenz

C Verlängerung der Dauer des Muskelsummenaktionspotenzials (MSAP)

D Reduktion der MSAP-Amplitude

E Verlängerung der distal motorischen Latenz

\section{Frage 3}

Welche supportiven Kriterien einer CIDP sind nicht Teil der EFNS/PNS Guideline?
A T2-Hyperintensitäten im MRT
B zytalbuminäre Dissoziation im Liquor
C demyelinisierende oder remyelinisierende Aspekte in der Nervenbiopsie
D positives Therapieansprechen
E multifokale Nervenschwellungen im Nervenultraschall

\section{Frage 4}

Bei welchen Neuropathien ist der N. suralis elektrophysiologisch eher nicht ausgespart?
A MMN
B axonal motorische Variante des Guillain-Barré-Syndroms (AMAN)
C klassische CIDP
D diabetische Polyneuropathie
E CISP

\section{Frage 5}

Bei welcher Neuropathie gehören Leitungsblöcke zu den Diagnosekriterien?
A CMT1
B diabetische Polyneuropathie
C Small-Fiber-Neuropathie
D MMN
E vaskulitische Polyneuropathie

\section{Frage 6}

Welche Erkrankung ist die häufigste chronische immunvermittelte Polyneuropathie?
A CIDP
B diabetische Polyneuropathie
C Small-Fiber-Neuropathie
D MMN
E vaskulitische Polyneuropathie

\section{Frage 7}

Was empfiehl die DGN-Leitlinie zur Behandlung der nicht systemischen vaskulitischen Polyneuropathie?
A Glukokortikoide oder Cyclophosphamid
B Vitamin-B-Präparate
C Alphaliponsäure
D Rituximab
E Ceftriaxon

\section{Frage 8}

Welche sonomorphologischen Befunde sind untypisch?
A Bei der MMN finden sich oft regional begrenzte Faszikelschwellungen.
B Die CIDP zeigt oft ein heterogenes Bild mit Betonung pro- ximaler Nervensegmente.
C Bei der Vaskulitis finden sich oft gering ausgeprägte, lokal begrenzte Nervenschwellungen.
D Patienten mit einer CMT1 zeigen sehr ausgeprägte Nervenschwellungen.
E Patienten mit paraproteinämischer Neuropathie zeigen keine Nervenschwellungen.




\section{Punkte sammeln auf CMIE.thieme.de}

Fortsetzung $\ldots$

\section{Frage 9}

Welche Aussage zur Therapie der MMN ist nicht richtig?
A Therapie der ersten Wahl sind regelmäßige Immunglobulingaben.

B In seltenen Fällen können Steroide hilfreich sein.

C Immunsuppressive Verfahren konnten bisher keine Evidenzklasse I erreichen.

E In Einzelfällen kann Cyclophosphamid erwogen werden.

F Immunglobuline scheinen sowohl intravenös als auch subkutan zu helfen.

\section{Frage 10}

Welche Aussage zu den paraproteinämischen Polyneuropathien ist richtig?

A Es finden sich IgG4-Antikörper gegen paranodale Strukturen.

B Die paraproteinämischen Polyneuropathien gehören zur vaskulitischen PNP.

C Die Abklärung eines multiplen Myeloms kann man sich sparen.

D Eine beim IgM-MGUS vorkommende Variante mit Anti-MAGAntikörpern ist von therapeutischer Relevanz.

E Patienten mit paraproteinämischer Neuropathie zeigen keine Nervenschwellungen. 